\title{
Intraoperative Hypertensive Urgency and Hemodynamic Instability during Microvascular Decompression for Trigeminal Neuralgia
}

\author{
Pushkaran Jayapaul $^{1}$ Thanga T. Rajan ${ }^{1} \quad$ Aravind Sabeson $^{2}$ \\ ${ }^{1}$ Saveetha Medical College Hospital, Thandalam, Chennai, \\ Tamil Nadu, India \\ 2 Stanley Medical College Hospital, Chennai, Tamil Nadu, India \\ Address for correspondence Pushkaran Jayapaul, MCh, Department of \\ Neurosurgery, Saveetha Medical College \& Hospital, Thandalam, Chennai \\ 602105, Tamil Nadu, India (e-mail: pushkaranneuro@gmail.com).
}

Indian J Neurosurg 2019;8:103-107

\begin{abstract}
Keywords

- microvascular decompression

- ectatic atheromatous vertebrobasilar artery

- intraoperative hypertensive urgency

Objective To evaluate the factors predisposing to rare events of intraoperative hypertensive urgency during microvascular decompression (MVD) for trigeminal neuralgia (TGN) and outcome of MVD on long-term blood pressure (BP) control.

Patients and Methods Total 54 consecutive TGN patients who failed medical management in two institutions from January 2012 to January 2017 were included in the study. MVD of TGN was done through retromastoid suboccipital craniotomy. Intraoperative "hypertensive urgency" and hemodynamic instability were observed in two patients with BP shooting up to $>180 / 120 \mathrm{~mm} \mathrm{Hg}$, which was controlled. Postoperative period was uneventful and patients were discharged. Patients were reviewed in the OPD clinic at 1 and 6 months after discharge and their clinical status was evaluated. The collected data were analyzed retrospectively.

Results Of the 54 patients, 32 (59.26\%) were male and 22 (40.74\%) were female. Twenty-five (46.30\%) patients were hypertensive, out of whom 19 (76\%) were in Joint National Committee (JNC) 7 stage 1 and 6 (24\%) were in stage 2. Two (3.70\%) patients had an intraoperative hypertensive crisis, and in both the patients, the offending vessel was an atheromatous ectatic vertebrobasilar artery. Postoperative long-term BP control or normalization was observed in one of the two patients with a single antihypertensive drug who was on three antihypertensive drugs preoperatively.

Conclusion Intraoperative hypertensive urgency is a medical emergency that can be encountered in MVD surgery for TGN, and an ectatic atheromatous vertebrobasilar arterial compression on the nerve is notorious for predisposing to hypertensive urgency. Postoperative long-term BP control in hypertensive patients is not feasible after MVD in this study, which needs further detailed analysis.
\end{abstract}

\section{Introduction}

As per the International Association for the Study of Pain (IASP), trigeminal neuralgia (TGN) is defined as "a sudden and usually unilateral severe brief stabbing recurrent pain in the distribution of one or more branches of the fifth cranial nerve." 1

It is usually an excruciating unilateral facial pain, lasting for $<2$ minutes, ${ }^{2}$ which occurs spontaneously or by a triggering factor such as gentle, innocuous stimuli and segregated by a pain-free interval of varying duration. The region of transition of central and peripheral myelination called the Obersteiner-Redlich zone or root entry/exit zone (REZ) is vulnerable to pathophysiologic changes due to vascular compression ( - Fig. 1) that results in demyelination and altered transmission. ${ }^{3,4}$

The peripheral pathogenetic mechanism of TGN is induced by progressive dystrophy in the peripheral branches of the trigeminal nerve, which can be evoked by the compression syndrome (-Fig. 2) (neurovascular compression received

September 28, 2018

accepted

November 2, 2018

published online

August 27, 2019
Dol https://doi.org/

$10.1055 / \mathrm{s}-0039-1695670$

ISSN 2277-954X.
C2019 Neurological Surgeons'

Society of India
License terms

(®) (1) $\circledast$ 


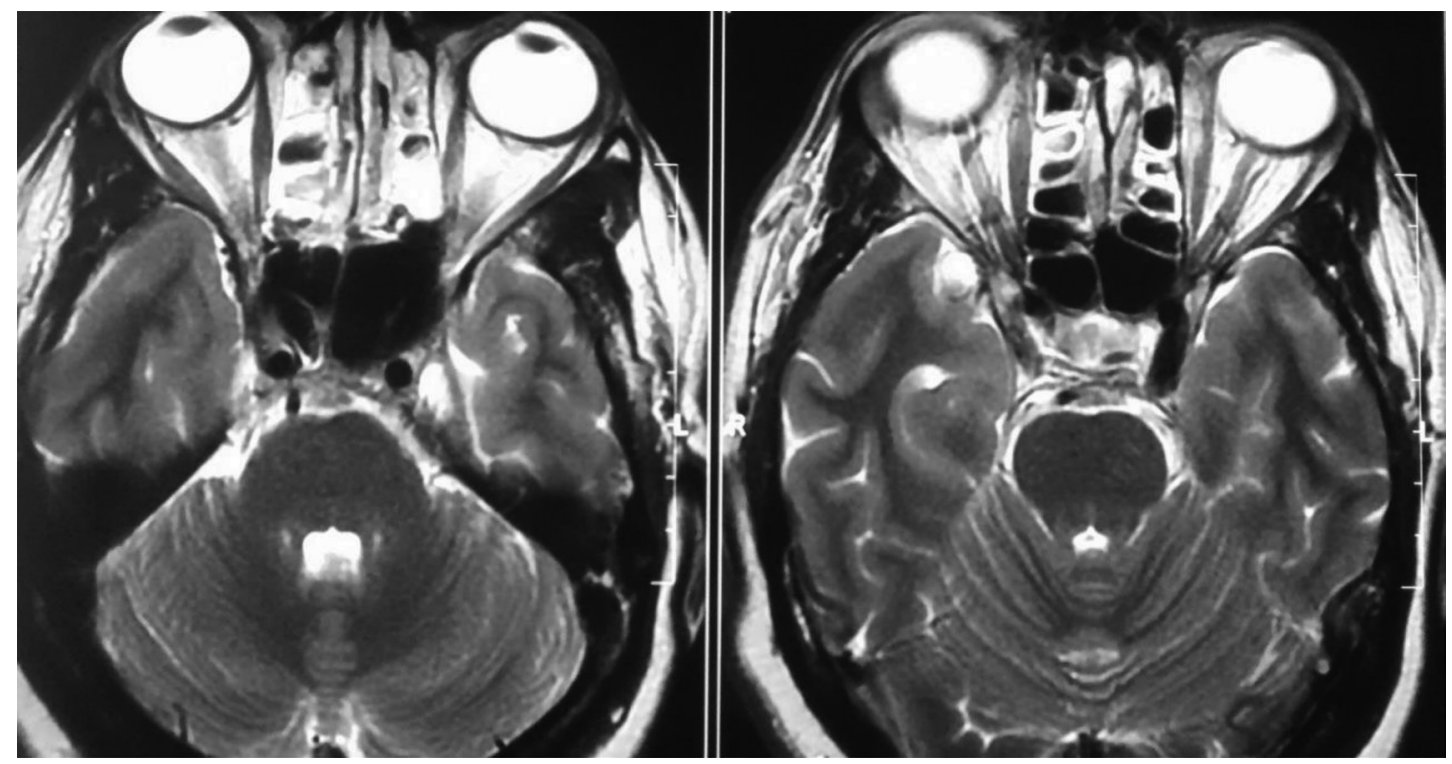

Fig. 1 T2-weighted MRI showing ectatic basilar artery in a TGN patient.

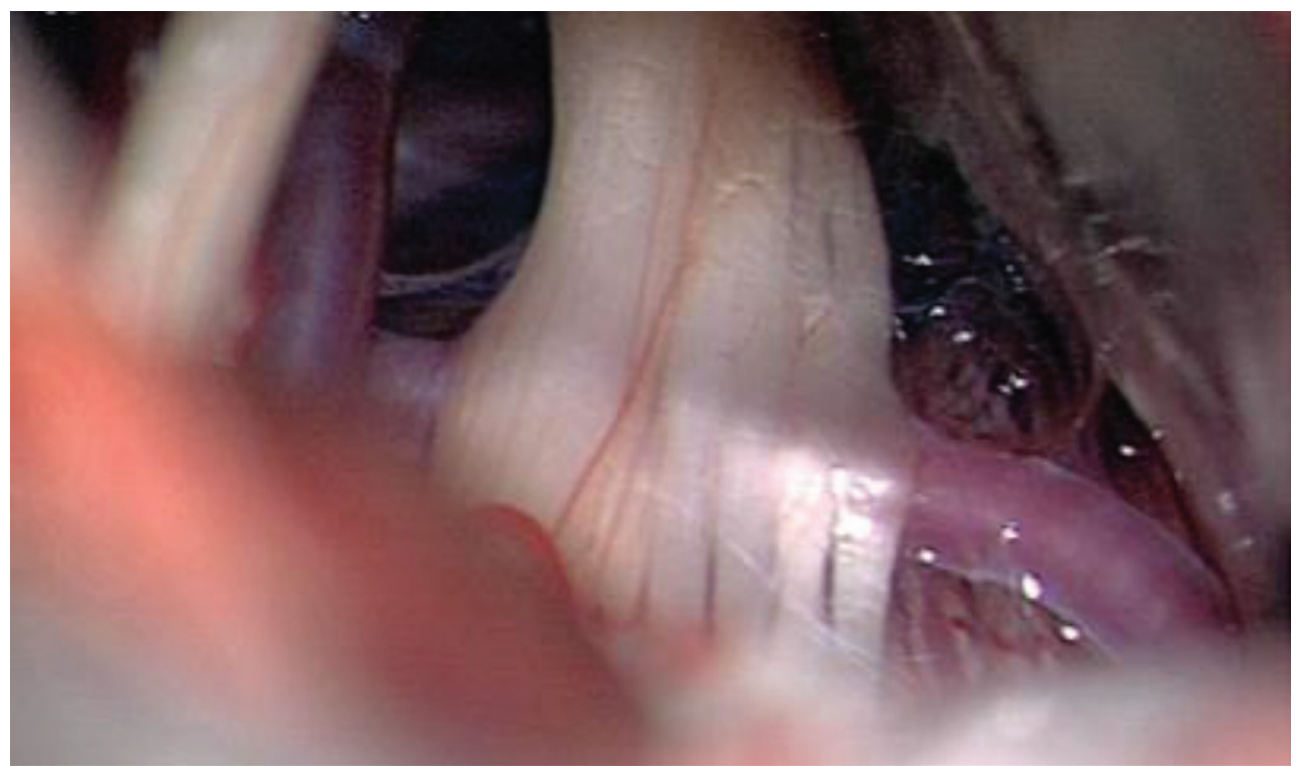

Fig. 2 Intraoperative picture showing neurovascular conflict in trigeminal neuralgia.

due to neoplasms, narrowed bone canals, and others) or allergic-immune reaction (mast cell degranulation and histamine release). This predetermines long-lasting afferent impulsation and formation of a central pathogenetic mechanism (a stable pathologic paroxysmal-type irritation focuses in the central nerve system $).{ }^{5}$ The pathologic substrate of this condition is mainly associated with demyelination of the trigeminal root entry zone. ${ }^{6}$

In this regard, experimental evidence exists about ectopic impulse generation from demyelinated axons. ${ }^{7}$ Accordingly, the pulsatile compression would result in progressive demyelination and subsequent ephaptic coupling, leading to aberrant impulse generation and spreading. ${ }^{3}$

Two cases of TGN developed intraoperative hypertensive urgency while undergoing microvascular decompression (MVD), and both cases had ectatic vertebrobasilar artery
(VBA) with atheromatous plaques compressing the trigeminal nerve. It was managed and postoperative period was uneventful. On long-term follow up, the blood pressure (BP) control was found to be satisfactory in one of the cases, whereas in the other patient, it was unchanged.

\section{Patients and Methods}

Fifty-four consecutive patients having medical refractory TGN who underwent MVD surgery from January 2012 to January 2017 in two separate institutions were included in this study.

\section{Surgical Technique}

Under general anesthesia, the patient laid in park bench position with affected side facing up and head fixed in three-pin 
Mayfield head rest. Retromastoid suboccipital craniotomy was done using craniotome. Dura was incised, followed by opening of subarachnoid cistern to drain cerebrospinal fluid (CSF). Cerebellum retracted minimally to reach the prepontine cistern. The neurovascular conflict was identified, and the thickened arachnoid layer was dissected. The offending vessel was mobilized from the nerve, and a thin Teflon patch was placed between them. Hemostasis was secured, dura sutured, bone flap replaced, and wound closed in layers.

\section{Perioperative Period and Follow-up}

Two patients developed intraoperative hypertensive urgency with BP shooting up to $>180 / 120 \mathrm{~mm} \mathrm{Hg}$, and in both cases, the offending vessel was an ectatic atheromatous VBA. In the event of occurrence of intraoperative hypertensive crisis in both the cases, the hemodynamics was stabilized by immediate cessation of manipulation of the vessel and temporary packing of the field, with anesthetic medications administering inhalational sevoflurane at $1 \mathrm{MAC}$ (minimum alveolar concentration) and propofol infusion at $10 \mathrm{mg} / \mathrm{min}$ intravenously. Following normalization of BP, surgery proceeded at a very slow pace with extreme extra caution and MVD was completed.

Immediate postoperative pain relief was achieved in all patients. Postoperative period was uneventful. Patients were discharged after routine postoperative care and reviewed in outpatient clinic after 1 and 6 months. Their clinical status were evaluated and recorded.

\section{Clinical Assessment}

The offending vessel on the trigeminal nerve and perioperative hemodynamic status of the patients were analyzed. BP and antihypertensive drug(s) dosage in the pre-, peri-operative, and follow-up period was noted.
Drastic fluctuation in intraoperative BP was considered for analysis. Postoperative BP status along with the requirement of antihypertensive medications for controlling systemic hypertension was compared with the patient's preoperative status.

\section{Statistical Analysis}

The collected data were analyzed retrospectively. Quantitative variables were presented as median (interquartile range) and non-normal distributions as mean. Standard deviation (SD) for normal distributions was assessed. Categorical variables were presented as absolute numbers and percentages. Pre- and postoperative BP comparisons were analyzed. A $p<0.05$ indicated statistical significance.

\section{Results}

Average age of patients at the time of surgery was 53.88 $( \pm 7.50)$ years. Thirty-two $(59.26 \%)$ patients were male and $22(40.74 \%$ ) were female ( - Fig. 3). Average duration of pain symptom was $2.3( \pm 0.77)$ years.

Twenty-five (46.30\%) patients were hypertensive and compliant to antihypertensive medications. As per Joint National Committee (JNC) 7 classification, 19 (76\%) patients were in JNC 7 stages 1 and 6 (24\%) were in JNC 7 stage 2.

Hemifacial pain was distributed along the segment(s) of the trigeminal nerve and the segment(s) involved were V2 in 13 (24.07\%) patients, V3 in 9 (16.66\%), V1V2 in 8 (14.81\%), and V2V3 in 24 (44.44\%) patients.

The offending vessel was superior cerebellar artery (SCA) in $29(53.70 \%$ ) cases, anterior inferior cerebellar artery (AICA) in 15 (27.77\%), petrosal vein (PV) in 7 (12.96\%), and ectatic VBA in 3 (5.55\%) cases (- Fig. 4).

\section{Gender distribution}

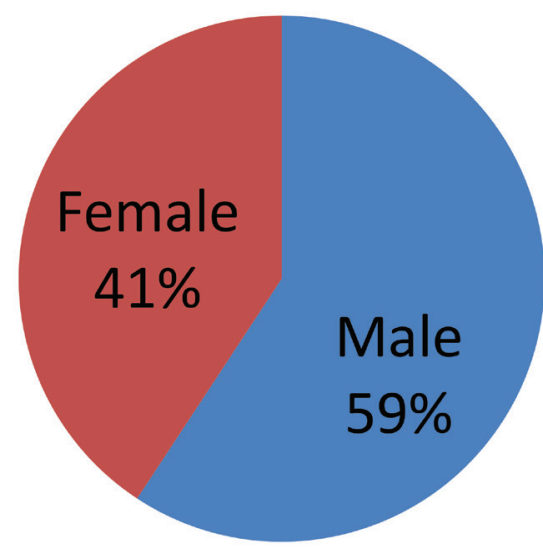

Fig. 3 Male and female distribution of TGN patients. 


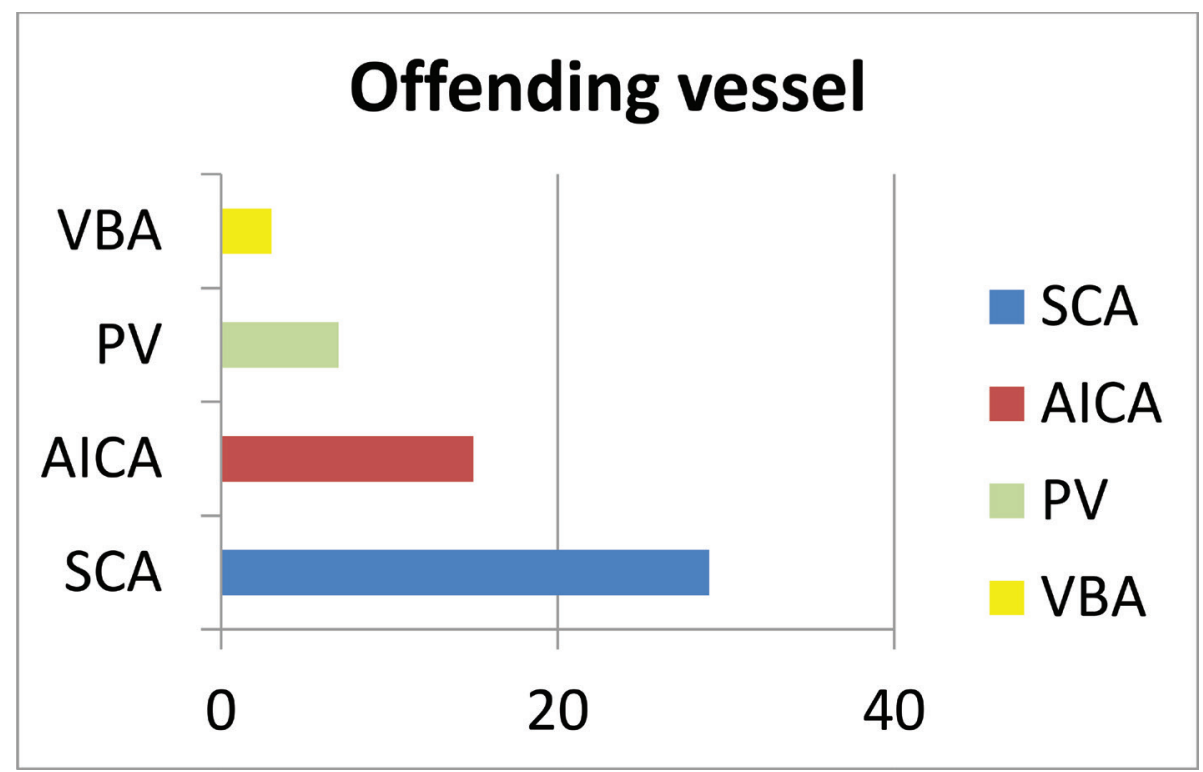

Fig. 4 Offending vessel compressing the trigeminal nerve and their numbers. AICA, anterior inferior cerebellar artery; PV, petrosal vein; SCA, superior cerebral artery; VBA, vertebrobasilar artery.

Table 1 Reported cases of vertebrobasilar artery compression on trigeminal nerve causing trigeminal neuralgia

\begin{tabular}{|l|l|l|l|}
\hline Study & Vertebrobasilar artery cases & Total cases studied & $\begin{array}{l}\text { Percentage of vertebrobasilar artery cases } \\
\text { studied (\%) }\end{array}$ \\
\hline Piatt et al & 2 & 105 & 1.90 \\
\hline Bederson et al & 4 & 256 & 1.56 \\
\hline Klun et al & 2 & 220 & 0.91 \\
\hline Honey et al & 13 & 552 & 2.4 \\
\hline
\end{tabular}

Intraoperative hypertensive urgency with BP > 180/120 $\mathrm{mm} \mathrm{Hg}$ was noted in 2 (3.70\%) cases when mobilizing the offending vessel from the nerve. It was found that in both the cases, the offending vessel was an ectatic atheromatous VBA. No drastic intraoperative hemodynamic instability was encountered in $52(96.30 \%)$ cases.

On follow-up of patients at 1 and 6 months after discharge, BP of 1 (4\%) patient out of the 25 hypertensive patients was found to be in good control. Preoperatively the patient was in JNC 7 stage 2 on three-drug regimen and postoperatively in JNC 7 stage 1 on monotherapy drug regimen. This patient had an intraoperative hypertensive urgency. The other patient who also had intraoperative hypertensive urgency was in JNC stage 1 preoperatively on a single antihypertensive drug, and it was unchanged in the follow-up period too. BP status of all other hypertensive patients was unchanged in the follow-up period. A Student $t$-test value of 0.848 signifies no correlation between MVD and postoperative long-term BP control/normalization.

\section{Discussion}

Vertebrobasilar dolichoectasia is an extremely rare entity with an expected incidence of 0.06-5.8\%. ${ }^{8}$ Direct compression by vertebrobasilar dolichoectasia is an uncommon cause for TGN. The incidence, as estimated in previous reports, ranges from 0.9 to $5.7 \%$. Piatt et al reported 2 cases in a series of 105 patients. ${ }^{9}$ Bederson et al reported 4 cases in a group of 256 operated cases. Klun et al reported 2 cases in a group of 220 operated patients. ${ }^{9}$ Honey et al, in their series of 552 patients who underwent MVD for TGN, reported 13 (2.4\%) patients with dolichoectatic vertebrobasilar compressions (10 males, 3 females $)^{10}$ (-Table $\mathbf{1}$ ). The incidence of recurrence after MVD surgery for VBA compression has been reported to range from 3 to $30 \% .{ }^{11}$ Mendoza and Illingworth reported that $90 \%$ of recurrences occurred within 2 years. ${ }^{12}$

"Hypertensive urgency" is the term suggested for patients with severe hypertension without acute end-organ damage, and "hypertensive crisis" is the term suggested for patients with severe hypertension with end-organ damage. ${ }^{13}$

There are reports on intraoperative hypotension due to trigeminocardiac reflex ${ }^{14,15}$ and even on long-term normalization/control of BP postoperatively after MVD. ${ }^{16,17}$

However, intraoperative hypertensive crisis during MVD in TGN is comparatively underreported. Israelyan et al in their study reported intraoperative hypertension during trigeminal nerve manipulation in 14.6 and $14.4 \%$ of patients in the semi-sitting position group and semi-lateral position group, respectively. ${ }^{18}$

In this retrospective study, we came across two events of intraoperative hypertensive urgency. In both the cases, the offending vessel was an atheromatous ectatic VBA (100\%).

The rostral ventrolateral medulla (RVLM) regulates tonic sympathetic activity and plays a critical role in baroreflex 
arcs. Current knowledge indicates that nerval BP regulation is determined by the balance of powerful tonic excitatory and inhibitory inputs. ${ }^{19}$ Stimulation of the trigeminal nerve causes a vasopressor response by activating the RVLM, an important brainstem vasomotor center. ${ }^{20,21}$ Furthermore, TNS has been shown to decrease cerebrovascular resistance via the trigeminal-cerebrovascular system. ${ }^{22}$ When activated, these pathways, via RVLM and trigeminal-cerebrovascular system, can lead to significant increase in cerebral perfusion, hence making the trigeminal nerve a promising target in TBI management. Sympathetic nerve activity and BP are temporary reduced by MVD in patients with severe hypertension with neurovascular compression. ${ }^{23}$ TGN is associated with essential hypertension. ${ }^{24}$

There are animal and human studies that hypothesize the compression on rostral ventral medulla (RVM) to be a cause of essential hypertension (neurogenic hypertension). MVD of the lower cranial nerves and RVM has been performed to control or normalize BP in essential hypertensive patients. ${ }^{25}$

\section{Conclusion}

Trigeminal neuralgia due to an ectatic atheromatous VBA is notorious for predisposing to intraoperative hypertensive urgency, and postoperative long-term BP control in hypertensive patients is not feasible after MVD.

\section{Conflict of Interest}

None.

\section{References}

1 Merskey H, Bogduk N. Classification of Chronic Pain. Descriptors of Chronic Pain Syndromes and Definitions of Pain Terms. 2nd ed. Seattle, WA: IASP Press; 1994

2 Neuralgia T. IHS Classification ICHD-II. International Headache Society. Available at: http://ihs-classification.org/en/02_klassifikation/04_teil3/13.01.01_facialpain.html. Accessed January 25, 2011

3 Grasso G, Landi A, Alafaci C. A novel pathophysiological mechanism contributing to trigeminal neuralgia. Mol Med 2016;22:452-454

4 Love S, Coakham HB. Trigeminal neuralgia: pathology and pathogenesis. Brain 2001;124(Pt 12):2347-2360

5 Sabalys G, Juodzbalys G, Wang H-L. Aetiology and pathogenesis of trigeminal neuralgia: a comprehensive review. J Oral Maxillofac Res 2013;3(4):e2

6 Jannetta PJ. Neurovascular compression in cranial nerve and systemic disease. Ann Surg 1980;192(4):518-525

7 Smith KJ, McDonald WI. Spontaneous and evoked electrical discharges from a central demyelinating lesion. J Neurol Sci 1982;55(1):39-47

8 Fonseka CL, Tissera WAJN. A case of trigeminal neuralgia due to dolichoectasia of the vertebrobasilar arteries. Galen Med J 2016;21(2):27-29

9 Luiz KJ, Filho P, Azambuja A, Gustavo de D, de Barros FM. Vertebrobasilar dolichoectasia as a cause of trigeminal neuralgia: the role of microvascular decompression. Case report.
Arq Neuro-Psiquiatr. [Internet]. 2006 Mar [cited Sep 14, 2018];64(1):128-131

10 Honey CM, Kaufmann AM. Trigeminal neuralgia due to vertebrobasilar artery compression. World Neurosurg 2018; 118:e155-e160

11 Lee SH, Levy EI, Scarrow AM, Kassam A, Jannetta PJ. Recurrent trigeminal neuralgia attributable to veins after microvascular decompression. Neurosurgery 2000;46(2):356-361, discussion 361-362

12 Mendoza N, Illingworth RD. Trigeminal neuralgia treated by microvascular decompression: a long-term follow-up study. $\mathrm{Br}$ J Neurosurg 1995;9(1):13-19

13 Christos V, Katsi V, Nihoyannopoulos P, Lekakis J, Dimitris T. Cardiovascular hypertensive crisis: recent evidence and review of the literature. Front Cardiovasc Med 2013;3:51

14 Schaller B. Trigeminocardiac reflex. A clinical phenomenon or a new physiological entity? J Neurol 2004;251(6):658-665

15 Sandu N, Chowdhury T, Sadr-Eshkevari P, et al. Trigeminocardiac reflex during cerebellopontine angle surgery: anatomical location as a new risk factor. Future Neurol 2015;10:7-13

16 Jannetta PJ, Segal R, Wolfson SK Jr. Neurogenic hypertension: etiology and surgical treatment. I. Observations in 53 patients. Ann Surg 1985;201(3):391-398

17 Geiger H, Naraghi R, Schobel HP, Frank H, Sterzel RB, Fahlbusch $R$. Decrease of blood pressure by ventrolateral medullary decompression in essential hypertension. Lancet 1998;352(9126):446-449

18 Israelyan L, Lubnin A, Shimanskiy V, Odamanov D. Neurosciences. The efficacy and complications of microvascular decompression of the trigeminal nerve in patients with trigeminal neuralgia according to a position of a patient during surgery: semilateral versus semisitting. Eur J Anaesthesiol 2014;31:111

19 Dampney RAL, Horiuchi J, Tagawa T, Fontes MAP, Potts PD, Polson JW. Medullary and supramedullary mechanisms regulating sympathetic vasomotor tone. Acta Physiol Scand 2003;177(3):209-218

20 McCulloch PF, Panneton WM, Guyenet PG. The rostral ventrolateral medulla mediates the sympathoactivation produced by chemical stimulation of the rat nasal mucosa. J Physiol 1999;516(Pt 2):471-484

21 Dutschmann M, Herbert $\mathrm{H}$. The medical nucleus of the solitary tract mediates the trigeminally evoked pressor response. Neuroreport 1998;9(6):1053-1057

22 Zhang QJ, Hara H, Kobayashi S. Distribution patterns of sensory innervation from the trigeminal ganglion to cerebral arteries in rabbits studied by wheat germ agglutinin-conjugated horseradish peroxidase anterograde tracing. Neurosurgery 1993 32:993-999, discussion 999

23 Frank H, Heusser K, Geiger H, Fahlbusch R, Naraghi R, Schobel HP. Temporary reduction of blood pressure and sympathetic nerve activity in hypertensive patients after microvascular decompression. Stroke 2009;40(1):47-51

24 Levy EI, Scarrow AM, Jannetta PJ. Microvascular decompression in the treatment of hypertension: review and update. Surg Neurol 2001;55(1):2-10,discussion10-11

24 van Ouwerkerk WJR, Samii M. Ammirati M, Essential hypertension in patients with hemifacial spasm or trigeminal neuralgia. In: Frowein RA, Brock M, Klinger M, eds. Head Injuries. Advances in Neurosurgery, vol 17. Berlin/Heidelberg, Germany: Springer; 1989

25 Levy EI, Scarrow AM, Jannetta PJ. Microvascular decompression in the treatment of hypertension: review and update. Surg Neurol 2001;55(1):2-10, discussion 10-11 